\title{
Efecto de la zona de procedencia y época de muestreo sobre la composición bromatológica de la torta de maracuyá
}

\author{
Effect of the area of origin and sampling time on the bromatological composition \\ of passion fruit cake
}

\author{
Edison Mazón-Paredes ${ }^{1 *}$ (D), Marcelino Herrera-Rodríguez ${ }^{2,5}$ (D), Carlos Mazón-Paredes ${ }^{3+}$ (D). Antón García-Martínez ${ }^{4}$ (D), \\ Manuel Delgado-Pertíñez ${ }^{5}$ (D) José Luis Guzmán-Guerrero 6 (iD)
}

\begin{abstract}
${ }^{1}$ Facultad de Ciencias Pecuarias, Universidad Técnica Estatal de Quevedo. Los Ríos, Ecuador. "2Instituto de Investigación y Formación Agraria y Pesquera (IFAPA). Cartaya, España. ${ }^{3}$ Facultad de Ciencias Pecuarias de la Universidad Técnica Estatal de Quevedo. Los

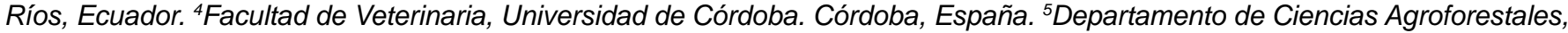
Universidad de Sevilla. Sevilla, España. ${ }^{6}$ Escuela Técnica Superior de Ingeniería, Departamento de Ciencias Agroforestales, "Campus de Excelencia Internacional Agroalimentario, ceiA3", Universidad de Huelva. Huelva, España.
\end{abstract}

Correo electrónico: emazon@uteq.edu.ec

\section{RESUMEN}

Como paso previo a la utilización de la torta de maracuyá (TM) en dietas prácticas en la alimentación de animales como fuente proteica en peces con el fin de abaratar su costo, se investigó el efecto de la zona de procedencia y la época de muestreo sobre su composición bromatológica. Para ello, se han utilizado dos muestras semanales al azar de 1.000 gramos, en agosto y septiembre, con dos repeticiones en cada una de las fábricas extractoras (Vinces y Guayas). El contenido de Materia Seca $(92,99 \pm 0,17 \%)$ y fósforo $(P)(0,33 \pm 0,01 \%)$ fueron superiores en Vinces y la Proteína Bruta (PB) $(23,38 \pm 0,29 \%)$ y la Fibra Bruta (FB) $(45,50 \pm 1,21 \%)$ inferiores. El contenido de PB $(24,61 \pm 0,27 \%)$ y FB $(51,20 \pm 0,27 \%)$ fueron superiores en la TM en septiembre y la Fibra Neutro Detergente $(71,80 \pm 0,34 \%)$ y el $P(0,31 \pm 0,01 \%)$ inferiores. Para el contenido en Extracto Etéreo $(12,02 \pm 0,29 \%)$, cenizas $(2,17 \pm 0,01 \%)$ y calcio $(3,11 \pm 0,07 \%)$ se encontró un valor superior para las muestras de agosto y en Vinces. Se concluye que la mayoría de los parámetros analizados de la TM dependen, tanto del lugar de procedencia como el mes de extracción. Lo que implica siempre un análisis bromatológico previo antes de ser usadas en alimentación animal.

Palabras clave: Passiflora edulis; composición bromatológica; mes de muestreo; zona de procedencia; subproducto

\section{ABSTRACT}

As a previous step to the use of passion fruit cake (PFC) in practical diets in animal feed as a protein source in fish in order to lower its cost, the effect of the area of origin and the time of sampling on its bromatological composition was investigated. For this, two weekly 1,000 grams samples from August and September were used with two repetitions for each of two extraction plants (Vinces and Guayas). The dry matter content $(92.99 \pm 0.17 \%)$ and phosphorus (P) $(0.33 \pm 0.01 \%)$ were higher in Vinces and Crude Protein (CP) $(23.38 \pm 0.29 \%)$ and Crude Fiber (CF) $(45.50 \pm 1.21 \%)$ were lower. The CP $(24.61 \pm 0.27 \%)$ and CF (51.20 $\pm 0.27 \%)$ content of PFC was higher in September and Neutral Detergent Fiber $(71.80 \pm 0.34 \%)$ and $P(0,31 \pm 0,07 \%)$ were lower. A higher value was found for Ether Extract (12.02 $\pm 0.29 \%)$, ash (2.17 $\pm 0.01 \%)$ and calcium $(3.11 \pm 0.07 \%)$ content in samples for August and Vinces. It is concluded that most of the PFC parameters analysed depend on both the place of origin and the time of extraction. This always implies the need for a prior bromatological analysis before its use in animal feed.

Key words: Passiflora edulis; bromatological composition; sampling season; area of origin; by-product 


\section{INTRODUCCIÓN}

El maracuyá (Passiflora edulis), originario de Brasil, fue introducido a finales de la década de los años 80 en Ecuador. Los cultivos de maracuyá se desarrollan especialmente en las zonas tropicales, como es el caso de las provincias de Esmeraldas, Manabí, Guayas, El Oro, Los Ríos y Santo Domingo de los Tsáchilas, alcanzando una producción de 40.274 toneladas (ton) de fruta en el Ecuador [3]. La fruta de color amarillo es la que más se cultiva y tiene una producción por hectárea (ha) de 10 a 25 ton. Tiene un alto nivel de rendimiento de jugo, pero la concentración de aroma es menor que la variedad roja [33]

Este producto tiene gran interés y aceptación en los Estados Unidos de Norteamérica (EUA) y en los países europeos, haciendo este cultivo muy prometedor y rentable en la economía ecuatoriana. Para el año 2017, la producción mundial alcanzó un estimado de 1,5 millones de ton [34]; Brasil, es el principal productor de parchita maracuyá, seguido de Colombia e Indonesia. En términos de exportación mundial en el 2017, Ecuador fue el primer productor en los mercados internacionales, seguido de Australia y Nueva Zelanda" [2]. Ecuador, durante el 2014, se ha situado como el mayor exportador de pulpa de maracuyá en el mundo, donde se destaca como principales consumidores a Holanda, EUA, Australia, Canadá, Portugal y Colombia [7].

La vida útil de esta planta es de 3 años (a) e inicia su etapa de producción de 8 a 12 meses (mes) [43], con lo que se garantiza una primera cosecha al a. Su fruto es fuente de proteínas, minerales, vitaminas, carbohidratos y grasas. Se consume como fruta fresca, se utiliza para preparar diferentes productos y se exporta principalmente como concentrados. La fruta de maracuyá contiene aproximadamente el $50,3 \%$ de cáscara, $23,2 \%$ de jugo y $26,2 \%$ de semillas [18]. El aceite que se extrae de su semilla puede ser utilizado para la fabricación de jabones, tintes y barnices; incluso antes de ser refinado puede ser utilizado en la alimentación de animales monogástricos. El maracuyá está disponible durante todo el a, con dos picos de producción: el primero de abril a junio y el segundo de agosto a octubre [24].

En los últimos quince a se han instalado en Ecuador varias fábricas dedicadas principalmente a procesar la pulpa del maracuyá. Éstas tienen por ventaja, la fácil adquisición de su materia prima debido a que en Ecuador hay destinada una gran superficie al cultivo de esta fruta; sin embargo, la marcada vulnerabilidad del precio de este producto, concentrado de maracuyá, en el mercado mundial eventualmente crea dificultades al sector [7].

El método de tratamiento y extracción de la torta de maracuyá (TM), se obtiene a partir de la semilla de la fruta de maracuyá, la cual previamente se le ha extraído el jugo. La semilla pasa por un proceso de secado primario para ser lavada y así eliminar el material mucilaginoso e impurezas, luego pasa al secado final en una secadora continua para finalmente ser molida. Presenta siete importantes etapas para la elaboración del producto, comenzando por la recepción del producto, el triturado, lavado, secado, molido, tamizado y, por último, el almacenamiento y distribución del mismo. Cada semilla de maracuyá rinde un $30 \%$ de aceite y un $67 \%$ de pasta conocida también como TM [6]. La mayoría de los trabajos publicados se refieren a la composición química de otros subproductos relacionados con la fruta de maracuyá como la semilla y la cáscara de maracuyá $[15,19,36]$.
El alto costo de los ingredientes tradicionales utilizados para la alimentación animal ha obligado a la búsqueda de nuevos alimentos y a la evaluación de su potencial alimenticio [21]. Uno de estos nuevos alimentos es la TM, un subproducto que no es bien conocido, pero con un gran potencial, por su alto contenido en proteína y fibra, en la alimentación de rumiantes [19] e incluso en no rumiantes [22, 31], y también debido a su costo muy bajo.

Existe un conocimiento limitado sobre la composición química y uso adecuado de la TM como alimento para animales, aspecto muy interesante ya que mediante estas materias primas alternativas podrían disminuirse los costos de alimentación en ganadería, que suponen más del $70 \%$ de los gastos totales [32]. Por tanto, el objetivo de este trabajo fue la caracterización bromatológica de la TM en distintas muestras recogidas en dos mes de muestreo (agosto y septiembre) y procedentes de las plantas extractoras de jugo de los cantones de Vinces y Guayas, como paso previo a su introducción en dietas alimenticias para el ganado.

\section{MATERIALES Y MÉTODOS}

\section{Características de las zonas de procedencia del subproducto utilizado}

El cantón Vinces tiene una superficie total de 58.925 ha. Su posición geográfica está ubicada en las coordenadas: $01^{\circ} 33^{\prime} 22^{\prime \prime} \mathrm{S}$ | $01^{\circ} 12^{\prime} 9^{\prime \prime} \mathrm{O}$, con una altura promedio de 30 metros sobre el nivel del mar (m.s.n.m). El relieve que cubre la zona central del cantón Vinces es básicamente plano, surcado a veces por lomas de poca altura, posee llanura baja con relieve moderado bajo, lomas bajas y redondeadas cuya altura no sobrepasa los 30 m.s.n.m. Son suelos profundos de cenizas recientes suaves y permeables. Presentan texturas francas limosas con arena muy fina; $\mathrm{pH}$ ligeramente acido a neutro; son ricos en materia orgánica y buena fertilidad natural y de coloración pardo rojizos. Su utilización es muy amplia soportando toda clase de cultivos, pastizales y arboledas [25].

La provincia del Guayas tiene una superficie total de 1.315.024 ha, su posición geográfica está ubicada en las coordenadas: $02^{\circ} 10^{\prime} \mathrm{S} \mid 79^{\circ} 54^{\prime} 60^{\prime \prime} \mathrm{O}$, con una altura promedio de 6 m.s.n.m. El clima en la zona de la provincia del Guayas es el resultado de la presencia de corrientes marinas, la corriente fría de Humboldt y la corriente cálida de Panamá, cuyos efectos varían a lo largo del a. La Provincia presenta los siguientes tipos de climas: tropical megatérmico árido a semi árido, tropical megatérmico seco a semihúmedo y tropical megatérmico húmedo [25].

\section{Localización y toma de muestras}

Para valorar la composición química de la TM se realizaron muestreos representativos al azar del proceso continuo de producción en cada una de las extractoras de aceite de este subproducto en los cantones de Vinces y Guayas, durante dos mes sucesivos en las dos procesadoras de fruta de maracuyá. Se tomaron dos muestras semanales de 1.000 gramos (g) de TM durante los mes de agosto y septiembre, con dos repeticiones por cada una de las procesadoras de fruta que fueron objeto de estudio, con un total de 64 muestras que fueron directamente analizadas. 


\section{Análisis químico-bromatológico del subproducto}

Los análisis químicos de las muestras se llevaron a cabo en los laboratorios de Santa Catalina del Instituto Nacional de Investigaciones Agropecuarias (INIAP), en Izabamba (0²1' S | 78³3'60" O; 3.058 m.s.n.m.), entre los meses de agosto y septiembre del 2013.

En la preparación de una muestra (unidad experimental) para el análisis se tuvieron en cuenta los siguientes procedimientos: cada muestra se mezcló cuidadosamente y se colocó sobre una superficie plana y siguiendo el método de los cuartos se tomaron porciones de los dos cuartos opuestos, se mezcló de nuevo y se repitió la operación las veces que fue necesario hasta obtener la cantidad deseada [40]. El peso final de la muestra para análisis fue de $1.000 \mathrm{~g}$ de TM. Las muestras se homogenizaron [40] y se pusieron en una funda plástica de cierre hermético y se identificaron. Previamente a su análisis, las muestras fueron trituradas y pasadas por un tamiz de 1 milímetro $(\mathrm{mm})$ de diámetro en un micro molino Wiley (marca Tecnal, modelo TE-648, High Tech Service S.A.C, Perú). Se determinaron la materia seca (método 934.01), las cenizas (método 942.05), el extracto etéreo (método 920.39), el nitrógeno (método 984.13) y la fibra bruta (método 978.10), de acuerdo con los métodos de la Asociación de Químicos Analíticos Oficiales (AOAC) [4]. Los valores de nitrógeno $(\mathrm{N})$ se determinaron por el procedimiento Kjeldahl [4], que convierte el $\mathrm{N}$ en proteína bruta mediante la multiplicación por el factor 6,25.

Los análisis de fibra neutro detergente (FND), fibra ácido detergente (FAD) y lignina ácido detergente (LAD) se llevaron a cabo de acuerdo con Van Soest y col. [44], y fueron expresados sin la ceniza residual. Todas las fracciones de fibra fueron analizadas en un Extractor Fibertec 1030 Hot (Tecator AB, Suecia). El contenido de grasa se midió por extracción con éter de petróleo (punto de ebullición, de 40 a $60{ }^{\circ} \mathrm{C}$ ) en una unidad de extracción Soxtec System 1040 (FOSS Tecator AB, Suecia). La energía bruta (EB) se determinó por medio de una bomba calorimétrica adiabática (modelo C5003, marca IKA Werke, Direct Industry, EUA). Para el cálculo de la energía metabolizable se tomó en consideración la metodología de las ecuaciones matemáticas [1, 23].

\section{Tratamiento estadístico}

Los resultados de la composición bromatológica de las muestras fueron analizados mediante un análisis ANOVA, usando el Modelo Lineal General (GLM) de IBM SPSS Statistics para Windows (versión 25.0; IBM Corp., Armonk, Nueva York, EUA): en el modelo se han considerado los factores fijos zona procedencia y mes de muestreo en la recogida y la interacción entre ambos. El modelo matemático se indica a continuación:

$$
Y_{i j k}=\mu+P_{i}+M_{j}+(P M)_{i j}+\varepsilon_{i j k}
$$

Donde:

$Y_{i j k}=$ Medición realizada en la "k-ésima" unidad experimental, colectada en la "j-ésima" mes de muestreo y en la "i-ésima" planta procesadora.

$\mu=$ constante común a todas las observaciones, referida como media general

$$
\begin{aligned}
& P_{i}=\text { Efecto de la "i-ésima" planta procesadora, } \mathrm{i}=1,2 \\
& M_{j}=\text { Efecto de la "j-ésima" mes de muestreo, } \mathrm{j}=1,2
\end{aligned}
$$

$\mathrm{PM}_{\mathrm{ij}}=$ Efecto de la interacción de la "i-ésima" planta procesadora con la "j-ésima" mes de muestreo

$\varepsilon_{\mathrm{ijk}}=$ Efecto de los factores no controlados en el experimento sobre la "k-ésima" unidad experimental, colectada en la "j-ésima" mes de muestreo y en la "i-ésima" planta procesadora.

En caso de efectos significativos de la interacción entre los factores principales, las medias de los diferentes grupos fueron sometidas a la comparación múltiple de promedios mediante la prueba HSD Tukey [9].

\section{RESULTADOS Y DISCUSIÓN}

En la TABLA I se presentan los resultados de composición química de la TM producida en Ecuador. Hay que indicar que existe una gran variabilidad en el contenido de nutrientes en relación con los datos encontrados en la literatura $[8,11,14,20,28,34,41$, $45,47]$. Esta puede ser explicada por diversas causas. Romero y col. [42] señalan que algunos factores ambientales (latitud, altitud, Iluvia, topografía, textura y estructura de los suelos) actúan indirectamente sobre los diferentes procesos fisiológicos de las plantas, mientras que otros lo hacen en forma directa (radiación solar, fotoperiodo, temperatura, agua y fertilidad del suelo), lo que podría explicar esta variabilidad nutricional. Según Chamorro y col. [10], también las variedades de la fruta de maracuyá presentan variabilidad en la composición bromatológica, pero en el presente trabajo de investigación no se puede atribuir a este factor, debido a que en ambas localidades se utilizó la misma variedad (amarilla).

Por otra parte, el sistema de riego también podría influir; el más frecuente es el goteo que se practica en $42 \%$ de los cultivos, mientras que la mayoría depende de la precipitación. Además, existen otras razones que hacen que las zonas donde se produce el maracuyá se origine una variabilidad bromatológica nutricional, entre estos motivos están: la existencia de diferentes híbridos sembrados, diversas edades de siembra, distintos tipos de problemas fitosanitarios y modos de fertilización, entre otros [16]. También, las prácticas de manejo vegetal como la aplicación de micronutrientes directamente en las flores y frutos estimulan el crecimiento y llenado de éstos [26].

Por otro lado, algunos autores como Duval [12] y Jordán y Casaretto [27] reportan que las Giberelinas (especialmente la GA3) influyen en la formación de flores femeninas, en la reducción del número y tamaño de las semillas aumentando el contenido de pulpa en las frutas, al igual que se cree que retrasa la velocidad de maduración de los frutos después de cosechados.

Los valores de materia seca (MS) de este estudio fueron similares a los encontrados por Nutril [35], Quicornac [41] y Martínez y col. [30]. Los valores para la PB, EE y cenizas fueron superiores a los encontrados por Nutril [35] y Quicornac [41] e inferiores a los especificados por Martínez y col. [30].

Los valores obtenidos en el presente trabajo fueron determinados en diferentes épocas y circunstancias, considerando que hubo variabilidad en el piso climático, la profundidad y algunos factores físicos y químicos del suelo (densidad aparente, y porosidad total). También influyó la variabilidad del contenido de materia orgánica, la misma que pudo haber favorecido a la fertilidad de los suelos en las distintas zonas estudiadas [5]. Además, se puede señalar que los resultados en las localidades investigadas arrojaron una variación de la fertilidad de los suelos debido al piso climático, 
TABLA I

Composición química de la torta de maracuyá (Passiflora edulis) según la procedencia (zona de Vinces o Guayas) y según el mes de muestreo

\begin{tabular}{|c|c|c|c|c|c|c|c|c|}
\hline \multirow{2}{*}{$\begin{array}{l}\text { Composición química } \\
(\%, \text { base MS })^{1}\end{array}$} & \multicolumn{2}{|c|}{ Procedencia (P) } & \multicolumn{2}{|c|}{ Mes (M) } & \multirow[b]{2}{*}{ EEM $^{2}$} & \multicolumn{3}{|c|}{ Significación ${ }^{3}$} \\
\hline & $\begin{array}{l}\text { Vinces } \\
n=16\end{array}$ & $\begin{array}{c}\text { Guayas } \\
n=16\end{array}$ & $\begin{array}{c}\text { Agosto } \\
n=16\end{array}$ & $\begin{array}{c}\text { Septiembre } \\
n=16\end{array}$ & & $\mathbf{P}$ & $\mathbf{M}$ & PxM \\
\hline Materia seca (\%) & 92,99 & 92,32 & 92,60 & 92,70 & 0,10 & $* *$ & NS & NS \\
\hline Materia orgánica (\%) & 97,90 & 97,94 & 97,95 & 97,89 & 0,02 & NS & NS & NS \\
\hline Proteína bruta (\%) & 23,38 & 24,52 & 23,29 & 24,61 & 0,26 & * & ** & NS \\
\hline Extracto etéreo (\%) & 11,97 & 11,64 & 11,54 & 12,07 & 0,12 & NS & * & $\star *$ \\
\hline ELN (\%) & 21,49 & 20,49 & 20,75 & 21,22 & 0,38 & $* * *$ & $\star * *$ & $\star \star *$ \\
\hline Fibra bruta (\%) & 45,50 & 47,03 & 41,33 & 51,20 & 0,90 & $\star * \star$ & $\star * *$ & $\star \star *$ \\
\hline FND (\%) & 72,81 & 72,14 & 73,14 & 71,80 & 0,26 & NS & $\star * *$ & NS \\
\hline FAD (\%) & 69,26 & 69,33 & 69,57 & 69,01 & 0,29 & NS & NS & NS \\
\hline LAD (\%) & 44,01 & 44,79 & 44,80 & 43,99 & 0,31 & NS & NS & NS \\
\hline Cenizas (\%) & 2,10 & 2,06 & 2,05 & 2,11 & 0,02 & $\star \star$ & $* * *$ & $\star * \star$ \\
\hline Calcio (\%) & 2,89 & 2,81 & 2,94 & 2,76 & 0,04 & NS & $* * *$ & $\star \star \star$ \\
\hline Fósforo (\%) & 0,33 & 0,31 & 0,33 & 0,31 & 0,00 & $\star \star \star$ & $\star *$ & $\star *$ \\
\hline $\mathrm{EB}\left(\mathrm{Mcal} \cdot \mathrm{Kg}^{-1} \mathrm{MS}\right)$ & 5,24 & 5,15 & 5,22 & 5,18 & 0,06 & NS & NS & NS \\
\hline EM (Mcal.kg-1MS) & 1,28 & 1,26 & 1,26 & 1,27 & 0,01 & NS & NS & NS \\
\hline
\end{tabular}

${ }^{1} \mathrm{MS}$ : materia seca; ELN: extracto libre de nitrógeno; FND: fibra neutro detergente; FAD: fibra ácido detergente; LAD: lignina ácido detergente; EB: energía bruta; EM: energía metabolizable. La EM fue calculada usando las siguientes ecuaciones [1, 23]: $\mathrm{EM}(\mathrm{KJ})=15,66 \times \mathrm{MOD}(\mathrm{g})$ and $\mathrm{MOD}(\%)=107,01-0,963 \times \mathrm{FAD}(\%$, DM); donde MOD es la materia orgánica digestible. ${ }^{2}$ EEM: error estándar de la media. ${ }^{3} \mathrm{NS}=$ no significativo $(\mathrm{P} \geq 0,05) ; * \mathrm{P} \leq 0,05 ; * \star \mathrm{P} \leq 0,01$; $* * * \mathrm{P} \leq 0,001$.

la profundidad y algunos indicadores físicos y químicos como el contenido de nutrientes, $\mathrm{pH}$ y el cambio del uso del suelo [8].

Además, probablemente las enmiendas de los suelos para satisfacer las necesidades de los cultivos produjeron consecuencias sobre el rendimiento y la calidad de la fruta [45]. El contenido de ELN obtenido en esta investigación fue inferior al obtenido por Luna [29] (44,93 \%). El valor obtenido de FB fue similar al registrado por Nutril [35], pero superior al encontrado por Martínez y col. [30] (37,7 \%) e inferior al registrado por Quicornac [41] $(58,35 \%)$. Además, en cuanto a los componentes de la pared celular, los valores obtenidos de FND de la presente investigación fueron superiores a los señalados por la Organización de las Naciones Unidas para la Agricultura y la Alimentación (FAO) [38] y Noguera y col. [34]. También el contenido de FAD fue superior al encontrado en los estudios realizados por Chamorro y col. [10] y Noguera y col. [34] con la variedad Passiflora maliformis, lo que pudo deberse al empleo de distinta variedad, distinta parte y a diferente edad de madurez de la fruta.

Al comparar los resultados de esta investigación en cuanto a cenizas, éstos fueron inferiores a los obtenidos por Noguera y col. [34] (7,9 \%) y superiores a los datos obtenidos por Pantoja [40] (1,70 \%). En cuanto al contenido medio de calcio de la TM fue alto $(2,85 \%)$ con relación al contenido que reporta Veliz [46] $(1,3 \%)$ y Espejo [13] $(0,5 \%)$, estudio realizado en la variedad flavicarpa y en una zona de alta precipitación. El contenido de fósforo $(0,32 \%)$ fue inferior al reportado por la Organización de las Naciones Unidas para la Alimentación y la Agricultura FAO [37] $(0,4 \%)$ y Espejo [13] (1,7\%); no obstante, Noguera y col. [34] encontraron valores más bajos $(0,21 \%$ de calcio y $0,15 \%$ de fósforo), aunque estos datos refieren a la cáscara de maracuyá.

Finalmente, en este estudio, el valor medio obtenido para la EB (5,20 Mcal $\left.\cdot \mathrm{Kg}^{-1} \mathrm{MS}\right)$ de la TM es algo inferior al encontrado por Fachinello y col. [17], pero en harina de semilla de maracuyá $\left(5,56 \mathrm{Mcal} \cdot \mathrm{Kg}^{-1} \mathrm{MS}\right)$. Y el valor de EM $\left(1,27 \mathrm{Mcal} \cdot \mathrm{Kg}^{-1} \mathrm{MS}\right)$ es también más bajo si se compara con el obtenido por Luna [29] $\left(2,85 \mathrm{Mcal} \cdot \mathrm{Kg}^{-1}\right.$ MS) aunque en cáscara de maracuyá.

Los valores de $M S$ y fósforo $(P \leq 0,01)$ fueron superiores en la TM procedente del área de Vinces, mientras que la $\mathrm{PB}$ y la FB, fueron superiores $(P \leq 0,05)$ en la TM procedente de Guayas. La variabilidad bromatológica de este subproducto, en las zonas 
de Vinces y Guayas, podría ser debido a la variación de las características del suelo, como apunta Espejo [13], en algunos lugares el suelo profundo y bien drenado, de textura franca y una buena retención de humedad, pudo influir en la calidad de la TM.

Romero y col. [42], señalan que los factores ambientales como la latitud, altitud, lluvia, topografía, textura y estructura de los suelos actúan indirectamente sobre los diferentes procesos fisiológicos de las plantas, mientras que otros lo hacen en forma directa como la radiación solar, fotoperiodo, temperatura, agua y fertilidad del suelo. Estos factores ambientales afectan la producción agrícola de los cultivos en general, entre ellos la fruta de maracuyá, disminuyendo el potencial productivo o desmejorando la calidad de la fruta fresca. Por lo tanto, las condiciones ambientales y edafológicas particulares de las zonas donde se realizó la investigación pudieron determinar diferentes características, las que afectaron el crecimiento, desarrollo y producción de los cultivos de maracuyá.

De acuerdo con el informe de Cañizares y Jaramillo [8], la fruta del maracuyá se desarrolla en zonas cálidas donde la temperatura varía entre 24 a $28{ }^{\circ} \mathrm{C}$ y con precipitaciones de hasta $1.500 \mathrm{~mm}^{3}$ anuales. Estas dos localidades están en dos zonas ecológicas distintas con diferentes rangos de temperatura y de precipitación. La zona central de Vinces es óptima para el cultivo del maracuyá ya que este cantón se encuentra en una localidad plana con pequeñas ondulaciones de bajo relieve que no sobrepasan los 30 m.s.n.m., con suelos profundos y reciente formación volcánica, textura franca limosa, $\mathrm{pH}$ ligeramente neutro, ricos en materia orgánica y buena fertilidad. En tanto que, la zona de Guayas posee diferentes climas desde el tropical megatérmico árido hasta el tropical megatérmico seco a húmedo. Estas fuentes de variación edáfica y climatológica posiblemente sean las causas para que hayan influenciado en la composición química y por ende del subproducto TM.
No obstante, para el resto de los parámetros analizados en este trabajo (MO, EE, FND, FAD, LAD, calcio, EB y EM) no se observaron diferencias $(P>0,05)$ en función del lugar de procedencia de la TM.

En cuanto al efecto del mes de muestreo se ha observado, que los valores de PB y FB fueron superiores $(P \leq 0,01)$ en la TM en septiembre, mientras que la FND y el fósforo fueron superiores $(P \leq 0,01)$ en agosto. Por el contrario, el mes de muestreo no ha afectado a los valores de MS, MO, FAD, LAD y EB y EM $(P \geq 0,05)$.

Se ha encontrado un efecto significativo en la interacción Procedencia-Mes $(P \leq 0,01)$ para los parámetros EE, ELN, FB, cenizas, calcio y fósforo (TABLAI).

Debido a la naturaleza de la interacción [39] para la FB y fósforo, se ha procedido a interpretar solamente el efecto de los factores principales (en párrafos anteriores). En la TABLA II se detalla la prueba de separación de las medias para cada uno de estos parámetros.

En cuanto a las demás interacciones, se pudo observar cómo las muestras tomadas en agosto tienen un mayor contenido de EE cuando proceden de la zona de Vinces $(P \leq 0,05)$, mientras que para las muestras en septiembre no ha habido diferencias significativas entre las dos zonas de procedencia ( $P \geq 0,05)$ (FIG. 1).

En cuanto al contenido de ELN, en las muestras tomadas en septiembre, el contenido fue superior en la zona de Vinces $(P \leq 0,05)$ mientras que, en las muestras tomadas en agosto, el contenido fue superior en Guayas ( $P \leq 0,05)$ (FIG. 2).

Para el contenido de cenizas en las muestras tomadas en septiembre, el contenido fue superior en la zona de Guayas $(P \leq 0,05)$ mientras que, en las muestras tomadas en agosto, el contenido fue superior en Vinces ( $P \leq 0,05)$ (FIG. 3).

TABLA II

Composición química de la torta de maracuyá según la zona de procedencia (Vinces o Guayas) y según el mes de muestreo

\begin{tabular}{|c|c|c|c|c|c|}
\hline \multirow{4}{*}{$\begin{array}{c}\text { Composición química } \\
(\%, \text { base MS }) 1\end{array}$} & \multicolumn{4}{|c|}{ Procedencia2 } & \multirow{4}{*}{ EEM3 } \\
\hline & \multirow{2}{*}{\multicolumn{2}{|c|}{$\begin{array}{c}\text { Vinces } \\
\text { Mes }\end{array}$}} & \multirow{2}{*}{\multicolumn{2}{|c|}{$\begin{array}{c}\text { Guayas } \\
\text { Mes }\end{array}$}} & \\
\hline & & & & & \\
\hline & $\begin{array}{l}\text { Agosto } \\
(n=8)\end{array}$ & $\begin{array}{c}\text { Septiembre } \\
(n=8)\end{array}$ & $\begin{array}{l}\text { Agosto } \\
(n=8)\end{array}$ & $\begin{array}{c}\text { Septiembre } \\
(n=8)\end{array}$ & \\
\hline Extracto etéreo (\%) & $12,02 a$ & $11,93 a$ & $11,07 \mathrm{~b}$ & $12,21 \mathrm{a}$ & 0,12 \\
\hline ELN (\%) & $19,18 \mathrm{c}$ & $23,79 a$ & $22,32 \mathrm{~b}$ & $18,65 \mathrm{~b}$ & 0,38 \\
\hline Fibra bruta (\%) & $40,84 d$ & $50,16 \mathrm{~b}$ & $41,82 \mathrm{c}$ & $52,24 a$ & 0,90 \\
\hline Cenizas (\%) & $2,17 a$ & $2,04 \mathrm{~b}$ & $1,93 \mathrm{c}$ & $2,18 a$ & 0,02 \\
\hline Calcio (\%) & $3,11 \mathrm{a}$ & $2,67 \mathrm{~b}$ & $2,77 \mathrm{~b}$ & $2,84 \mathrm{~b}$ & 0,04 \\
\hline Fósforo (\%) & $0,36 a$ & $0,31 \mathrm{~b}$ & $0,31 \mathrm{~b}$ & $0,31 \mathrm{~b}$ & 0,01 \\
\hline
\end{tabular}

${ }^{1} \mathrm{MS}$ : materia seca; ELN: extracto libre de nitrógeno. ${ }^{2}$ Medias con diferente letra, en la misma fila indican diferencias significativas $(P \leq 0,05)$. ${ }^{3} E E M$ : error estándar de la media 


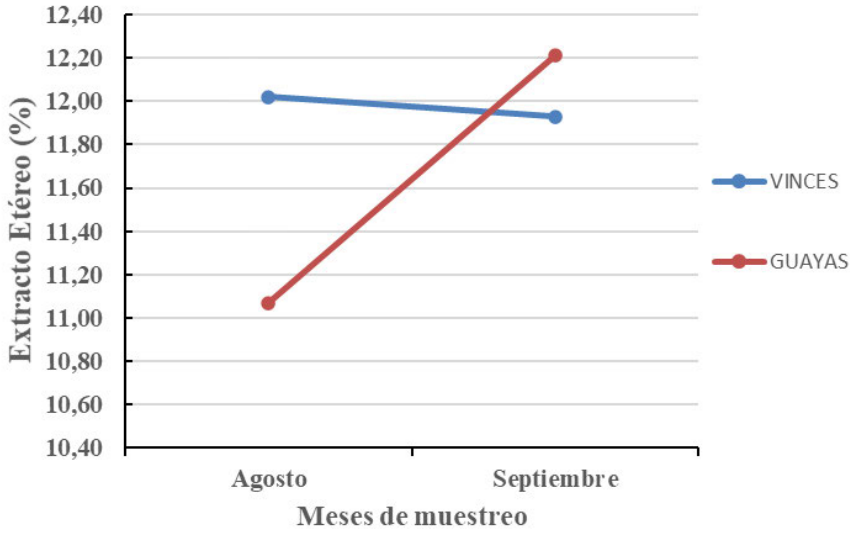

FIGURA 1. Efecto de la interacción de la zona de procedencia (Vinces o Guayas) y mes de muestreo (agosto y septiembre), en el contenido de extracto etéreo de la torta de maracuyá

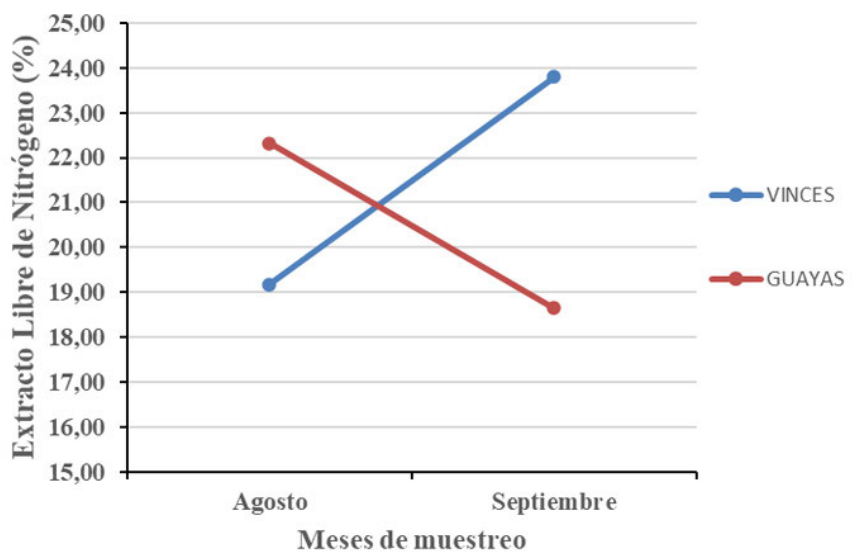

FIGURA 2. Efecto de la interacción de la zona de procedencia (Vinces o Guayas) y mes de muestreo (agosto y septiembre), en el contenido de sustancias extractivas libres de Nitrógeno de la torta de maracuyá

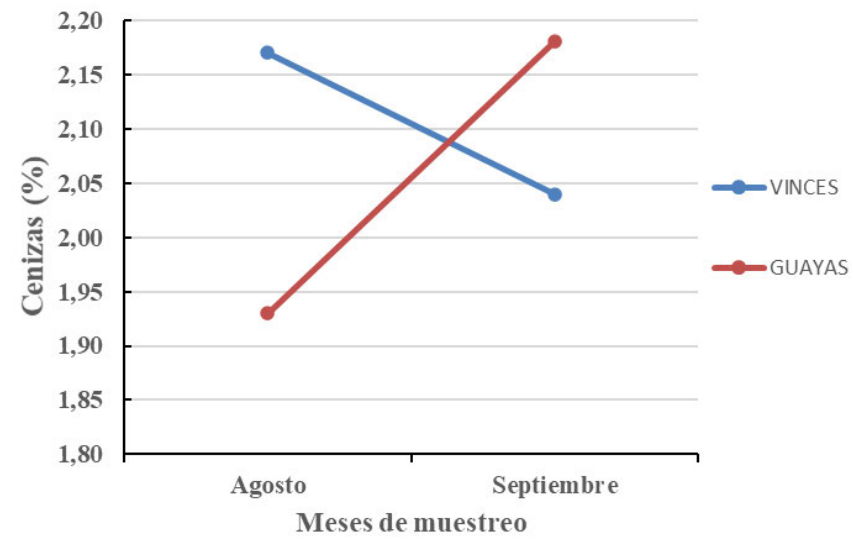

FIGURA 3. Efecto de la interacción de la zona de procedencia (Vinces o Guayas) y mes de muestreo (agosto y septiembre), en el contenido de cenizas de la torta de maracuyá

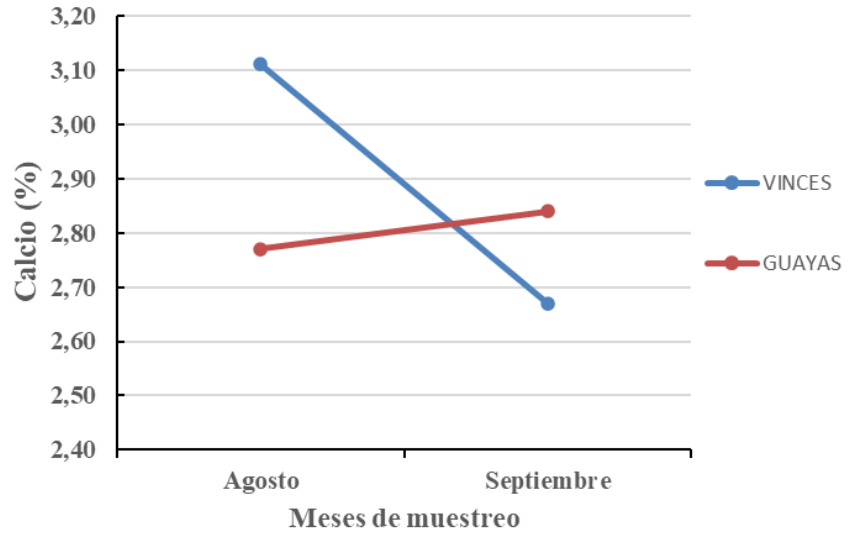

FIGURA 4. Efecto de la interacción de la zona de procedencia (Vinces o Guayas) y mes de muestreo (agosto y septiembre), en el contenido de Calcio de la torta de maracuyá

En cuanto al contenido en calcio se observó que el valor fue superior para las muestras procedentes de Vinces en agosto $(P \leq 0,05)$, mientras que para septiembre no se observan diferencias significativas entre ambas procedencias $(P \geq 0,05)$ (FIG.4).

\section{CONCLUSIONES}

La zona de procedencia de la TM influyó sobre el contenido de MS y fósforo, siendo los valores superiores en el área de Vinces, mientras que los contenidos de PB y FB fueron superiores en las muestras procedentes de Guayas. No obstante, la zona de procedencia no influyó en el contenido bromatológico de la TM para la MO, EE, FND, FAD, LAD, EB y EM.

El mes de muestreo también afectó al contenido de PB y FB, siendo los valores superiores en la TM recogida en septiembre, mientras que la FND y el fósforo fueron superiores en agosto. Sin embargo, no hubo efectos sobre los valores de MS, MO, FAD, LAD, EB y EM.

El contenido de EE, cenizas y calcio de la TM fue diferente en cada época de recogida en función de su procedencia; de tal forma que se encontró un valor superior para las muestras formadas en agosto y en la zona de Vinces.

La TM, por su gran variabilidad en la composición bromatológica según su zona de procedencia y mes de muestreo, debe ser analizada antes de ser utilizada en alimentación animal.

\section{AGRADECIMIENTOS}

Esta investigación tuvo la ayuda del Departamento de Posgrado de la Universidad Técnica Estatal de Quevedo, Ecuador y de INIA-FSE. 


\section{REFERENCIAS BIBLIOGRÁFICAS}

[1] AGUILERA, J. Aportaciones al conocimiento de la nutrición energética de pequeños rumiantes, con particular referencia al ganado caprino. Arch. Zoot. 50: 565-596. 2001.

[2] ALTENDORF, S. Frutas Tropicales Menores: Incorporación de un nicho de mercado. Organización de las Naciones Unidas para la Alimentación y la Agricultura (FAO). 2018. En línea: https://bit.ly/3BPrHSw. 22.07.2019.

[3] ARREAGA, L. La producción y exportación de las principales frutas no tradicionales y su importancia en las exportaciones totales del Ecuador, periodo 2012-2016". Universidad de Guayaquil. Tesis de Grado. Pp 35-36. 2016.

[4] ASSOCIATION OF OFFICIAL AGRICULTURAL CHEMISTS (AOAC). Official Methods of Analysis. 14th Ed. Assoc. Off. Anal. Chem. Arlington. 1141 pp. 2005.

[5] BRAVO, C.; RAMIREZ, A.; MARIN, H.; TORRES, B.; ALEMAN, R.; TORRES, R; NAVARRETE, H.; CHANGOLUISA, D. Factores asociados a la fertilidad del suelo en diferentes usos de la tierra de la Región Amazónica Ecuatoriana. Rev. Electrón. Vet. 18 (11): 1-16. 2017.

[6] CALLE, A.; COBOS, L. Estimación de consumo de aceite esencial de maracuyá. Escuela Politécnica del Litoral. Tesis de Grado. Pp 58-60. 2005.

[7] CALLE, Z.; GUARIGUATA, E.; GIRALDO, E.; CHARÁ, J. La producción de maracuyá (Passiflora edulis) en Colombia: perspectivas para la conservación del hábitat a través del servicio de polinización. Intercien. 35(3): 207-212. 2010.

[8] CAÑIZARES, A.; JARAMILLO, E. Propiedades nutricionales y usos. El cultivo de la maracuyá en Ecuador. Universidad Técnica de Machala, Ediciones UTMACH. 25 pp. 2015.

[9] CERVANTES, A.; MARQUEZ, M. Diseño de Experimentos. Curso Práctico. Universidad Autónoma de México. Publicado con apoyo de los proyectos PAPIME EN216403 y EN203503. Delegación Iztapalapa, junio 11 al 15, ciudad de Iztapalapa. México, D.F. 9 pp. 2007.

[10] CHAMORRO, D.; GONZÁLEZ, J.; COTRINO, A.; MORENO, J. Evaluación química y predicción de bovinos de ceba suplementados con pericarpios de Passiflora edulis I. y Passiflora maliformiss en sistemas silvopastoriles. Universidad Nacional Abierta y a Distancia. Tesis de Grado. Pp 1-4. 2007.

[11] DUCHI, N.; PAZMIÑO, J. Informe Final Proyecto IQ-CV-24, Convenio de Investigación ESPOCH-PROMSA. Riobamba, Ecuador. 120 pp. 2002.

[12] DUVAL, R. Hormonas Vegetales: Crecimiento y desarrollo de las plantas. Rev. Hort. 196 (2): 22-27. 2006.

[13] ESPEJO, C. Producción de maracuyá. 2016. Centro de Estudios Universidad de San Martin de Porres. Facultad Ciencias Administrativas y Recursos Humanos. En línea: https://bit.ly/3E8I4Nc. 05.03.2016.

[14] ESPINOZA, I. Características fermentativas y nutritivas de ensilajes de forrajes tropicales con diferentes niveles de inclusión de residuos agroindustriales de cáscara de maracuyá (Passiflora edulis). Córdoba, España. Tesis Doctoral Pp 34-35. 2015.
[15] ESPINOZA, I.; MEDINA, M.; BARRERA, A.; MONTENEGRO, L.; SANCHEZ, A.; ROMERO, M. Composición bromatológica y degradabilidad ruminal in situ de residuos agroindustriales de maracuyá (Passiflora edulis) y plátano (Musa paradisiaca). Cien. Tec. UTEQ. 10(2): 63-67. 2017.

[16] ESTUPIÑAN, G.; SANTACRUZ, L.; CRISTANCHO, A. Caracterización de las variables de crecimiento, niveles foliares y de rendimiento en dos materiales genéticos de palma OxG y DxP en diferentes edades de desarrollo de la plantación Guaicaramo S.A. Rev. Palmas. 34: 99-108. 2013.

[17] FACHINELLO, M.; POZZA, P.; FURLAN, A.; PAULA, V.; BONAGURIO, L.; MARCATO, S.; LEAL, I.; HUEPA, L. Nutritional evaluation of passion fruit seed meal for meat quails. Rev. Bras. Saúde Prod. Anim. 17 (2): 202-213. 2017.

[18] FERRARI, R.; COLUSSI, F.; AYUB, R. Caracterização de subprodutos da industrialização do maracujá - Aproveitamento das Sementes. Rev. Bras. Frutic. 26 (1): 101-102. 2004.

[19] FILIAN, W.; SALINAS, J.; ARIAS, R.; GÓMEZ, J. Evaluación físico-química de residuos agroindustriales para la alimentación animal. J. Sci. Res. 5 (1): 1-7. 2020.

[20] FREIRE, D. Evaluación de un balanceado inicial en base a pasta de maracuyá, en terneros y terneras de biotipo lechero hasta el período de destete, en el cantón Quero Provincia de Tungurahua. Universidad de las Américas. Tesis de Grado. 27 pp. 2016.

[21] GAIBOR, W. Evaluación del efecto de la adición de cuatro niveles de cáscara de maracuyá y polvillo de arroz en la dieta alimenticia para el acabado de novillos en estabulación. Santo Domingo de los Tsáchilas, Ecuador. Tesis de Grado. 4 pp. 2013.

[22] GARAVITO, R; LÓPEZ, R. Alimentación de cerdos durante la fase de ceba con subproductos de maracuyá y otros frutos procesados. Cenicafé. 53 (3): 178-192. 2002.

[23] GASA, J.; CASTRILLO, C. Criterios de utilización de subproductos agroindustriales en la alimentación de rumiantes. Ministerio de Agricultura, Pesca y Alimentación, Madrid. Hojas divulgativas 13/91. 24 pp. 1992.

[24] HEREDIA, C. Análisis de criterios comerciales y financieros de maracuyá (Passiflora edulis fluvicarpa) en la provincia de El Oro. Universidad Técnica de Machala. Tesis de Grado. Pp 25-26. 2016.

[25] INSTITUTO NACIONAL DE HIDROLOGÍAY METEOROLOGÍA (INAMHI). Anuario Meteorológico No. 52. República del Ecuador. Quito, Ecuador. Pp 90 - 100. 2013.

[26] JARAMILLO, V.; CARDENAS, R.; OROZCO, A. Importancia alimentaria y usos. Manual sobre el cultivo de maracuyá (Passiflora edulis) en Colombia. Corporación Colombiana de Investigación Agropecuaria-CORPOICA. 80 pp. 2009.

[27] JORDAN, M.; CASARETTO, J. Hormonas y Reguladores del Crecimiento: Auxinas, Giberelinas y Citocininas. En: Fisiología Vegetal. Squeo, F, A.; Cardemil, L. (Eds.). La Serena: Ediciones Universidad La Serena. Pp 1-28. 2006.

[28] LQARI, H.; VIOQUE, J.; PEDROCHE, J; MILLAN, F. Lupinus angustifolius protein isolates: chemical composition, functional properties and protein characterization. Food Chem. 76: 349-356. 2002. 
[29] LUNA, G. Obtención de balanceado a partir de los desechos del maracuyá (Passiflora edulis variedad Flavicarpa) para ganado vacuno. Universidad Central del Ecuador. Universidad Central del Ecuador. Quito. Tesis de Grado. 81 pp. 2014.

[30] MARTÍNEZ, J; MEDINA, O; ZAMBRANO, R. Estudio fisicoquímico funcional de los aislados proteicos en semillas de maracuyá (Passiflora edulis f). Bistua. 9 (1): 70-76. 2011.

[31] MAZÓN, C; GUZMÁN, JL; GARCíA, A; MAZÓN, M; DELGADO, M; HERRERA, M. Digestibilidad aparente de dietas con harina de semillas de maracuyá sobre el desempeño productivo del pez nativo vieja azul (Aequidens rivulatus) en la etapa de cría. Rev. Ecuat. Invest. Agrop. 2 (2): 36-43. 2017.

[32] MEDINA, H; MARTÍNEZ, M; BONILLA, J. Caracterización bromatológica de materias primas y subproductos en el municipio Quibdo, Chocó. Rev. Instit. Universidad Tecnológica del Chocó. 26 (2): 9-12. 2007.

[33] MENA, C; BILSBORROW, R; MCCLAIN, M. Socioeconomic drivers of deforestation in the Northern Ecuadorian Amazon. J. Environ. Manage. 37(6): 802-815. 2006.

[34] NOGUERA, R; VALENCIA, S; POSADA, L. Efecto de diferentes aditivos sobre la composición y el perfil de fermentación del ensilaje de cáscaras de Maracuyá (Passiflora edulis). Livest. Res. Rural Dev. 26(9): 1-5. 2014.

[35] NUTRIL. Reporte químico bromatológico del Departamento de Nutrición y Alimentación Animal. Guayaquil, Ecuador. 29 pp. 1992.

[36] ONOFRE, J. Parámetros productivos en pollos criollos alimentados con torta de maracuyá (Passiflora edulis) como sustituto de la alimentación base. Universidad Técnica Estatal de Quevedo. Tesis de Grado. Pp 36-37. 2017.

[37] ORGANIZACIÓN DE LAS NACIONES UNIDAS PARA LA ALIMENTACIÓN Y LAAGRICULTURA (FAO). Manuales para el control de calidad de los alimentos. Introducción a la toma de muestras de alimentos. 1989. En línea: https://bit.ly/3jQXNao. 04.04.2018.

[38] ORGANIZACIÓN DE LAS NACIONES UNIDAS PARA LA ALIMENTACIÓN Y LAAGRICULTURA (FAO). Compendio de Agronomía Tropical. Editado por el Instituto Interamericano de Cooperación para la Agricultura y el Ministerio de Asuntos Extranjeros de Francia. 1998. San José de Costa Rica.
[39] OTT, RL; LONGNECKER, M. Chapter 15. Analysis of Variance for Standard Designs. In: An introduction to Statistical Methods and Data Analysis. 5th Ed. Crockett, C. Donnelley, R. R. \& Sons, Inc./Willard (Eds.)., USA. Pp 853-942. 2001.

[40] PANTOJA, A.; MURILLO, A.; MARTÍNEZ, H. Caracterización de aceite de semillas de maracuyá (Passiflora edulis Sims.) procedentes de residuos agroindustriales obtenido con $\mathrm{CO}_{2}$ supercrítico. Acta Agron. 66 (2): 178-185. 2016.

[41] QUICORNAC. Reporte del Análisis Químico de la Torta de Maracuyá. Empresa Procesadora de Frutas. Guayaquil, Ecuador. 7 pp. 2004.

[42] ROMERO, H; AYALA, I; RUIZ, R. Ecofisiología de la palma de aceite. Rev. Palmas. 28: 176-184. 2007.

[43] VALAREZO, A.; VALAREZO, O.; MENDOZA, A.; ÁLVAREZ, H.; VÁSQUEZ, W. EI Cultivo de maracuyá: Manual técnico para su manejo en el litoral ecuatoriano. 2014. Programa de Fruticultura Estación Experimental Portoviejo. Portoviejo, Manabí, Ecuador. En línea: http://repositorio.iniap.gob.ec/ handle/41000/1159. 07.04.2015.

[44] VAN SOEST, P; ROBERTSON, J; LEWIS, B. Methods for dietary fiber, neutral detergent fiber ad nonstarch polysaccharides in relation to animal nutrition. J. Dairy Sci. 74: 3582-3597. 1991.

[45] VARGAS, J; BENÍTEZ, D; TORRES, V; RÍOS, S; SORIA, S; NAVARRETE, H; PARDO, D; TORRES, A. Tipificación de fincas ganaderas de doble propósito en la provincia de Pastaza. Rev. Amazon. Cien. Tecnol. 3:183-197. 2014.

[46] VELIZ, A. Caracterización química y estabilidad aeróbica de tres variedades de cáscara de maracuyá. Quevedo, Los Ríos. Universidad Técnica Estatal de Quevedo. Tesis de Grado. Pp 15-16. 2017.

[47] VIGOR. Reporte de Análisis bromatológico de la torta de maracuyá del Departamento de Nutrición y Alimentación Animal. Guayaquil. Ecuador. 20 pp. 1992. 